\title{
Automedicação em idosos de um Programa Saúde da Família, Brasil
}

\section{Self-medication in the elderly in Family Health Program, Brasil}

\section{ABSTRACT}

In the elderly population, studies show the prevalence of use of prescription and consumption of medications without prescription from a licensed health care professional (self-medication), which leads to the potential for interactions with prescription drugs, adverse reactions and drug interaction. The present study aimed to identify the prevalence of self-medication, the determining factors in this practice, and the main drugs consumed without prescription in the elderly people. This paper describes cross-sectional study that evaluated self-medication practiced by elderly, who were assisted at the Strategy Health Family of São Luís/MA. Of the 100 elderly studied $63 \%$ was female and was $37 \%$ male; $72 \%$ reported using at least one medication during the days preceding the survey. Of these $33 \%$ reported exclusive use of prescribed drugs and $67 \%$ reported simultaneous use of prescribed and not prescribed. The most used non-prescribed drugs were analgesics (46,15\%) and anti -inflammatory $(22,31 \%)$. The most cited reasons for the practice of self-medication were general pain (65.26\%) followed by fever (16,26\%) and flu (7.37\%). Pharmaceutical assistance should be provided as a priority to the elderly, to avoid the misuse of medicines and ensure access to the correct drugs.

Key Words: Self-medication. Medication use. Elderly health.

\section{RESUMO}

Na população idosa, estudos apontam a predominância do uso de medicamentos prescritos, bem como consumo de medicamentos sem prescrição de um profissional de saúde habilitado (automedicação), o que leva a potenciais riscos de interações com os medicamentos prescritos, reações adversas e intoxicações. O presente estudo teve como objetivo identificar a prevalência da automedicação, os fatores determinantes nesta prática, e os principais medicamentos consumidos sem prescrição médica em uma população de idosos. Estudo transversal, com amostra aleatória simples realizada em uma Unidade de Estratégia de Saúde da Família de São Luís/MA. Foram entrevistados 100 idosos (63\% do sexo feminino e $37 \%$ do sexo masculino), onde $72 \%$ referiram uso contínuo de pelo menos um medicamento, desses $33 \%$ relataram consumo exclusivo de medicamentos prescritos e $67 \%$, uso simultâneo de prescritos e não prescritos. Os medicamentos sem prescrição mais utilizados foram os analgésicos (46,15\%) e os anti-inflamatórios (22,31\%). As causas mais citadas para a prática de automedicação foi à dor $(65,26 \%)$, seguida da febre $(16,26 \%)$ e gripe $(7,37 \%)$. Verificou-se que há grande prevalência da automedicação neste grupo, sendo os analgésicos os mais utilizados e a dor é o sintoma mais relatado no que concerne à automedicação. Deste modo, a atenção farmacêutica deve ser considerada uma das prioridades no atendimento aos idosos, com intuito de garantir o acesso adequado aos medicamentos e o uso racional desses.

Palavras-Chave: Automedicação. Uso de medicamentos. Saúde do idoso 


\section{INTRODUÇÃO}

O envelhecimento populacional vem ocorrendo de forma acentuada em países em desenvolvimento como consequência do aumento da expectativa de vida, da redução da fecundidade e da mortalidade infantil (1). É considerado um fenômeno mundial e configura como uns dos maiores desafios da saúde pública contemporânea (2).

Em 2020 os idosos, no Brasil, poderão totalizar $13 \%$ da população. Ainda segundo especialistas, o país, em 2025, será o sexto do mundo em números de pessoas na terceira idade, o que demanda cuidados especiais com essa população (3). A esperança de vida ao nascer deverá atingir em 2041 os 80 anos, chegando há 82 anos em 2060; sendo que no Maranhão essa expectativa deverá chegar aos 74 anos em 2030 (4).

Com o aumento da população idosa novos desafios surgem aos serviços e profissionais de saúde, pois o envelhecimento acomete órgãos e tecidos, elevando a prevalência de doenças crônicas não transmissíveis (hipertensão arterial sistêmica, doenças osteoarticulares, diabetes mellitus, entre outras), que demandam acompanhamento contínuo, exames periódicos e tratamento medicamentoso de uso prolongado $(5,6)$. As causas de adoecimento e morte neste grupo específico são de etiologia multifatorial e funcional o que se soma a utilização de grande variedade e disponibilidade de especialidades farmacêuticas $(7,8)$.

Os medicamentos representam um dos itens mais importante à saúde do idoso e necessitam de atenção especial (7). A automedicação (utilização de medicamentos sem prescrição) é extremamente comum e se constitui como um importante fator de risco para a saúde dos idosos $(3,9)$, devido às peculiaridades fisiológicas que representam essa população como alterações de massa corporal, diminuição da proporção de água, diminuição das taxas de excreção renal e do metabolismo hepático, as quais influenciam na eliminação do metabólico, no acúmulo de substâncias tóxicas no organismo e na produção de reações adversas (10).

Estudos mostram que a média de medicamentos utilizados por estes indivíduos é de dois a cinco por dia $(11,10)$ e que a iatrogenia tem sido apontada como um importante problema de saúde pública, uma vez que as interações medicamentosas são nocivas ao organismo humano, sobretudo no idoso (12).

Considerando a importância que representa o uso de medicamento na população idosa, principalmente a prática da automedicação, o presente estudo teve como objetivo identificar a prevalência da automedicação, os fatores determinantes nesta prática, e os principais fármacos consumidos sem prescrição médica na população de idosos atendidos em Programa de Estratégia de Saúde da Família (ESF) de São Luís, Maranhão.

\section{MÉTODO}

Estudo descritivo transversal, não probabilístico, realizado no município de São Luís, MA. A pesquisa foi desenvolvida na unidade da ESF nº. 118, da Unidade Básica de Saúde (UBS) Dr. Antônio Guarané, localizada no bairro do Coroadinho, São Luís/MA, a qual na sua área de cobertura abrange 300 indivíduos acima de 60 anos.

A pesquisa guiou-se pelos princípios éticos e legais da pesquisa em seres humanos seguindo as recomendações do Conselho Nacional de Saúde (CNS) conforme a Resolução $n^{\circ} 466$, de 2012, que trata das diretrizes e normas de pesquisa envolvendo seres humanos, sendo aprovado pelo Comitê de Ética do Hospital Universitário da Universidade Federal do Maranhão/HU/UFMA sob o protocolo n. 289937

Foi selecionada uma amostra aleatória simples, de ambos os sexos, sem distinção de etnia e patologia existente. A coleta de dados foi realizada por meio da aplicação de um questionário semiestruturado em consulta farmacêutica individual realizada nos meses de agosto a novembro de 2013 em dias aleatórios no turno matutino. Como critérios de inclusão foi adotado ter idade igual ou superior a 60 anos, com capacidade cognitiva preservada. Como critério de exclusão adotou-se portadores de transtornos mentais com dificuldades para o entendimento dos objetivos da pesquisa e do Termo de Consentimento Livre e Esclarecido (TCLE), acamados e impossibilitados de frequentarem a UBS.

Foi caracterizado como automedicação o consumo do medicamento sem a devida indicação por escrito do profissional prescritor ou indicado por outrem (conhecidos, parentes, amigos, vizinhos, entre outros) ou ainda o reaproveitamento de terapias anteriores. As variáveis, estudadas foram idade, sexo, escolaridade, renda per capita, situação previdenciária, problemas de saúde, medicamentos em uso, causas da automedicação, uso de medicação sem prescrição, classe dos medicamentos, indicação, frequência da automedicação e frequência da assistência médica.

Para identificação dos medicamentos foi solicitado que o voluntário apresentasse a embalagem do medicamento e/ou à prescrição médica para diminuir eventuais erros na anotação dos dados pelo entrevistador. Para identificar as substâncias a partir dos nomes comerciais, empregou-se o Dicionário de Especialidade Farmacêutica (DEF) (2012/2013) (13). Para apreciação dos dados utilizou-se tabelas, por meio do programa Microsoft Excel 2010 e Programa SPSS 18 (Statistical Package for the Social Sciences) e, posteriormente, a análise foi realizada através da estatística descritiva. 


\section{RESULTADOS E DISCUSSÃO}

A amostra foi composta por 100 idosos, dos quais $58 \%$ encontravam-se na faixa etária de 60 a 69 anos, sendo que $63 \%$ eram do sexo feminino, $47 \%$ relataram serem donas de casa e a maioria dos entrevistados possuía renda de até um salário mínimo (63\%) (Tabela 1).

Tabela 1: Perfil da população idosa entrevistada, segundo variáveis sócio demográficas, São Luís, MA, 2013

\begin{tabular}{|c|c|c|}
\hline VARIÁVEIS & $\mathbf{N}$ & $\begin{array}{c}\text { PREVALÊNCIA } \\
(\%)\end{array}$ \\
\hline \multicolumn{3}{|l|}{ Sexo } \\
\hline Feminino & 63 & 63 \\
\hline Masculino & 37 & 37 \\
\hline \multicolumn{3}{|l|}{ Faixa etária (anos) } \\
\hline $60-69$ & 58 & 58 \\
\hline $70-79$ & 36 & 36 \\
\hline 80 a mais & 6 & 6 \\
\hline \multicolumn{3}{|l|}{ Profissão } \\
\hline Dona de casa & 47 & 47 \\
\hline Lavrador & 14 & 14 \\
\hline Agente de saúde & 6 & 6 \\
\hline Outros & 33 & 33 \\
\hline \multicolumn{3}{|l|}{ Situação Conjugal } \\
\hline Solteiro & 20 & 20 \\
\hline Casado/União estável & 53 & 53 \\
\hline Viúvo & 22 & 22 \\
\hline Divorciado & 5 & 5 \\
\hline \multicolumn{3}{|l|}{ Aposentado } \\
\hline Sim & 77 & 77 \\
\hline Não & 23 & 23 \\
\hline \multicolumn{3}{|l|}{ Escolaridade } \\
\hline Analfabeto & 26 & 26 \\
\hline Ensino fundamental incompleto & 53 & 53 \\
\hline Ensino fundamental completo & 9 & 9 \\
\hline Ensino médio completo & 12 & 12 \\
\hline \multicolumn{3}{|l|}{ Renda per capta $\left(\mathrm{SM}^{*}\right)$} \\
\hline 0 até $1 / 2$ & 11 & 11 \\
\hline $1 / 2$ até 1 & 63 & 63 \\
\hline 1 até 2 & 21 & 21 \\
\hline$>2$ & 5 & 5 \\
\hline
\end{tabular}

*SM= Salário mínimo $(\mathrm{R} \$ 678,00)$
A prevalência do uso contínuo de medicamentos foi de $72 \%$. Entre os idosos que utilizam medicamentos, $33 \%$ relataram ter consumido aqueles que foram exclusivamente prescritos por médicos ou dentistas e 7,46\% referiram consumo simultâneo de medicamentos prescritos e não prescritos.

Em relação à automedicação, $67 \%$ dos idosos afirmaram que já realizaram esta prática em algum momento (últimos quinze dias que antecederam a participação no estudo), dentre os quais $56,71 \%$ se encontravam na faixa etária de 60 a 70 anos. A prevalência da automedicação foi maior no sexo feminino $(68,65 \%)$ do que no masculino $(31,35 \%)$. Foi verificado que entre os participantes que declararam realizar a automedicação, $62,69 \%$ possuíam renda familiar de até um salário mínimo e a maioria possui apenas o ensino fundamental incompleto $(53,74 \%)$ (Tabela 2$)$.

Tabela 2: Prevalência de automedicação na população idosa entrevistada, segundo sexo, idade, escolaridade, problemas de saúde e uso de medicamentos, São Luís, MA, 2013.

\begin{tabular}{|l|c|}
\hline \multicolumn{1}{|c|}{ VARIÁVEIS } & $\begin{array}{c}\text { AUTOMEDICAÇÃ } \\
\%(\mathbf{N}=67)\end{array}$ \\
\hline Sexo & \\
\hline Feminino & 68,65 \\
\hline Masculino & 31,35 \\
\hline Idade & \\
\hline 60 a 69 & 56,71 \\
\hline 70 a 79 & 35,82 \\
\hline 80 ou mais & 7,47 \\
\hline Escolaridade & \\
\hline Analfabeto & 26,86 \\
\hline Ensino fundamental incompleto & 53,74 \\
\hline Ensino fundamental completo & 5,97 \\
\hline Ensino médio completo & 13,43 \\
\hline Problemas de saúde & \\
\hline Sim & 85,07 \\
\hline Não & 14,93 \\
\hline Uso de medicamento continuo & \\
\hline Sim & 70,15 \\
\hline Não & 29,85 \\
\hline
\end{tabular}

Os medicamentos mais citados na automedicação pelos idosos foram os analgésicos e os anti-inflamatórios com $46,15 \%$ e $22,31 \%$, respectivamente. Em relação à indicação do medicamento $37,04 \%$ relataram 
utilizar por conta própria seguido de 17,28\% que exercem a automedicação por indicação de algum familiar. Quanto ao tempo da utilização do medicamento e leitura da bula, $35 \%$ o consumiram apenas em um dia e $28,4 \%$ assumiram que não possuem o hábito de ler a bula do medicamento, respectivamente.

Entre os motivos que levam os idosos a automedicação $39,24 \%$ referiram conhecimento e uso prévio do medicamento, $20,25 \%$ declararam falta de tempo para buscar profissionais e serviços de saúde e $16,46 \%$ por indicação de um conhecido. Entre os sintomas que levaram ao consumo do medicamento por conta própria, os mais citados foram à dor $(65,26 \%)$, e febre $(16,84 \%)$. Sendo que, dos idosos que se automedicaram $8,96 \%$ relataram problemas relacionados ao uso do medicamento, mas sem notificação adequada ao sistema de saúde.

Em relação à frequência do uso de medicamentos sem prescrição médica, evidenciou-se que $92,54 \%$ dos idosos fazem quando têm algum tipo de queixa clínica. E quanto à procura pelos serviços de saúde nos últimos dias, apenas $8,96 \%$ dos idosos que se automedicam referiram ter comparecido a menos de quinze dias a uma semana em consulta médica (Tabela 3 ).

\section{DISCUSSÃO}

A prevalência do uso de ao menos um medicamento não prescrito nos quinze dias que antecederam a pesquisa foi alta (67\%) entre os idosos do Programa de ESF. Independentemente do grau de desenvolvimento socioeconômico a automedicação constitui uma prática universal presente nas mais diversas sociedades e culturas (3). Os resultados deste estudo mostram que a maioria dos idosos entrevistados realiza a automedicação, mesmo fazendo uso de medicações prescritas, e esses dados corroboram com outros estudos realizados no país, que relatam que esta é uma prática constante em torno de 30 a $80 \%(2,3,6,14)$. A baixa conscientização sobre os riscos da automedicação e as dificuldades de acesso aos serviços de saúde são visto como explicações a está prática entre os idosos (2).

Os dados deste estudo apontam que o uso de medicamento não prescrito é mais comum entre as mulheres, sendo também descrito em outros trabalhos $(3,15$, 16). A maior tendência das mulheres em se automedicar pode ser explicada pelo papel que a mesma representa na sociedade, pois a figura da mulher está relacionada com o papel social, preocupação com a saúde da família, ter maior acesso às farmácias e, consequentemente, aos medicamentos de venda livre (17); mas deve-se destacar o maior percentual de mulheres voluntárias que participaram desta pesquisa.
Foi verificado que o fenômeno da automedicação não se difundiu igualmente em todas as faixas etárias, resultados esses que se assemelham ao encontrado por outros autores, onde os idosos a partir de 80 anos praticavam menos automedicação, possivelmente pela maior necessidade de utilização dos serviços de saúde $(18,19)$ ou pela existência de um cuidador de saúde para este indivíduo.

Tabela 3: Prática da automedicação nos idosos entrevistados, segundo sintomas, tipo de medicamentos, motivos e indicação para automedicação, São Luís-MA, 2013.

\begin{tabular}{|l|r|}
\hline \multicolumn{1}{|c|}{ VARIÁVEIS } & N (\%) \\
\hline Principais sintomas & \\
\hline Dor & $62(65,26 \%)$ \\
\hline Febre & $16(16,84 \%)$ \\
\hline Gripe & $7(7,37 \%)$ \\
\hline Diarreia & $3(3,16 \%)$ \\
\hline Outros & $7(7,37)$ \\
\hline Medicamento & \\
\hline Analgésico & $60(46,15 \%)$ \\
\hline Anti-inflamatório & $29(22,31 \%)$ \\
\hline Vitaminas & $15(11,54 \%)$ \\
\hline Outros & $26(20 \%)$ \\
\hline Motivos para automedicação & \\
\hline Conhecimento e uso prévio do medicamento & $31(39,24 \%)$ \\
\hline Falta de tempo para buscar profissionais de saúde & $16(20,25 \%)$ \\
\hline Indicação de um conhecido & $13(16,46 \%)$ \\
\hline Falta de recursos financeiros & $8(10,13 \%)$ \\
\hline Outros & $11(13,92 \%)$ \\
\hline Indicação para a automedicação & \\
\hline Conhecimento próprio & $30(37,04 \%)$ \\
\hline Familiares & $14(17,28 \%)$ \\
\hline Balconista & $8(9,88 \%)$ \\
\hline Propaganda & $5(6,17 \%)$ \\
\hline Outros & $24(29,63 \%)$ \\
\hline
\end{tabular}

A automedicação foi mais frequente entre os entrevistados com menor grau de escolaridade; porém esses também são a maioria dos voluntários. No Distrito Federal. 67\% dos idosos que relataram se automedicar, possuíam o ensino fundamental incompleto, sendo estes resultados semelhantes ao encontrados em Goiânia 61,7\% $(20,2)$. Porém, para Musial et al. (2007), o público que mais se utiliza da automedicação são pessoas com maior nível de escolaridade. $\mathrm{O}$ autor explicou que quanto maior a 
escolaridade, mais aptas as pessoas se julgam capazes de entenderem a medicação e consequentemente praticar a automedicação. Mas deve-se levar em conta que a dificuldade de acesso aos serviços de saúde contribui significativamente para a automedicação, principalmente quando se tem experiências positivas com as medicações ou há indicação de alguém da família (21).

Entre os medicamentos mais utilizados por automedicação, estão os analgésicos e os anti-inflamatórios. Dados semelhantes foram obtidos por Almeida et al (2012), onde $85 \%$ entre os idosos entrevistados praticavam a automedicação, principalmente com analgésico e anti-inflamatório (15). Vários estudos também têm indicado os analgésicos como os medicamentos mais empregados na automedicação $(3,14,19,22)$. O alto consumo de analgésico entre idosos pode ser explicado pela elevada prevalência de inflamação e dor, sintomas comuns nessa fase da vida, decorrentes principalmente das doenças crônicas (2).

Sobre a automedicação foi observado que a maioria dos idosos a pratica, sem influencia de outra pessoa. Dado semelhante foi encontrado por Galato et al (2012), que verificaram a familiaridade que o indivíduo tem a respeito do seu problema de saúde e as experiências positivas anteriores com os medicamentos têm influência significativa na prática da automedicação (16).

Assim, é possível que experiências positivas ou utilização prévia de medicamentos, na população estudada, seja entendida como justificativa para uma prática sem prejuízos a saúde, o que não condiz com a realidade. Pois, o uso concomitante de tais medicamentos com outros prescritos, associado a fatores intrínsecos do indivíduo e seu estado de saúde, requer avaliação dos riscos à saúde, especialmente no que tange às intoxicações e interações medicamentosas, estas últimas de grande relevância na saúde pública mundial (19). O aconselhamento farmacêutico pode e deve ser utilizado como um instrumento de educação terapêutica para o uso racional de medicamentos.

A dor foi o principal problema de saúde citado para o manejo da automedicação. Estudos realizados em países desenvolvidos e em desenvolvimentos tem mostrado que o hábito da automedicação está associado à presença de sinais e sintomas menores como a dor e febre, porém doenças ou condições crônicas levam ao maior consumo de medicamento prescrito $(3,15)$. Ressalta-se ainda que não somente a dor física leva a automedicação, mas também a psíquica, dado esse ressaltado por Souza, que destaca que a busca de alivio ou cura de algum desconforto físico ou mental estimulam a automedicação nos idosos (23).
Dentre os idosos que afirmaram se automedicar, 8,96\% relataram apresentar problemas relacionados ao uso do medicamento, dados condizentes também foram observados por Galato et al (2012), que encontraram prevalência de $6,4 \%$ na população estudada. Porém, não houve qualquer notificação dessas reações adversas ao uso de medicação ou contato com o serviço de saúde para constatação dos mesmos, o que configura-se como uma fragilidade da obtenção de dados fidedignos sobre a prevalência de reação adversa e problemas relacionados ao medicamento com automedicação na população entrevistada (16).

Deve-se ressaltar que a automedicação é um elemento do autocuidado, mas deve ser realizada de forma responsável, a fim de não causar prejuízos à saúde. Destaca-se ainda a importância de praticar a educação em saúde de maneira contínua com os profissionais da área da saúde, para que os mesmos se tornem multiplicadores de informações quanto ao uso racional de medicamentos, e também com os usuários dos serviços de saúde para que possam se tornar elementos chave no autocuidado.

Nesse contexto, a orientação do farmacêutico, enquanto profissional com competência para realizar aconselhamento sobre os medicamentos, é de fundamental importância e tem como um dos princípios a promoção de saúde e minimizar a interação medicamentosa, bem como a exposição do indivíduo a riscos de saúde desnecessários (19).

\section{CONCLUSÃO:}

Os resultados do estudo mostram que, apesar de ser um risco a saúde, a automedicação tem alta prevalência entre os idosos. Também apontam que entre os medicamentos mais utilizados estão os analgésicos para mitigar a dor, fato este que pode ser relacionado ao fácil acesso a esse tipo de medicamento, às farmácias domiciliares e às condições de saúde desta população. Diante da importância que se reveste o tema, o estudo vem somar a outros já realizados no Brasil no sentido de enfatizar a necessidade de promoção do uso racional de medicamentos neste segmento populacional, enfatizando a prática da atenção farmacêutica, contemplando a promoção e a educação em saúde.

\section{AGRADECIMENTOS}

Aos voluntários da pesquisa e a equipe da Estratégia de Saúde da Família da Unidade Básica de Saúde Dr. Antônio Guanaré. 


\section{REFERÊNCIAS}

1. Coelho Filho JM, Marcopito LF, Castelo A. Perfil de utilização de medicamentos por idosos em área urbana do Nordeste do Brasil. Rev Saúde Pública, São Paulo, 2004. 38(4): 557-64.

2. Santos TRA, Lima DM, Nakatani AYK, Pereira LV, Leal GS, Amaral RG. Consumo de medicamentos por idosos, Goiânia, Brasil. Rev Saúde Pública, São Paulo, 2013. 47(1): 94-103.

3. Barros JAC, Oliveira MPB, Sá MB. Automedicação em idosos na cidade de Salgueiro-PE. Rev Bras Epidemiol. 2007. 10(1):75-85.

4. IBGE. Instituto Brasileiro de Geografia Estáticas. [Internet]. 2013 [acesso em 2013 ago 30]. Disponível em:< $\mathrm{http}: / /$ saladeimprensa.ibge.gov.br/ noticias?view=noticia \&id=1\&busca=1\&idnoticia $=2455>$.

5. Lima-Costa MF, Veras R. Saúde pública e envelhecimento. Cad. Saúde Pública. 2003. 19(3):700-701.

6. Cascae EA, Falchetti ML, Galato D. Perfil da automedicação em idosos participantes de grupos da terceira idade de uma cidade do sul do Brasil. Arq Catarinenses Med. 2008. 37(1): 63-69.

7. Flores VB, Benvegnu LA. Perfil de utilização de medicamentos em idosos da zona urbana de Santa Rosa, Rio Grande do Sul, Brasil. Cad Saúde Pública. 2008. 24(6): 1439-46.

8. Ribeiro AQ, Rozenfeld S, Klein CH, César CC, Acurcio FA. Inquérito sobre uso de medicamentos por idosos aposentados, Belo Horizonte, MG. Rev Saúde Pública. 2008. 42(4):724-32.

9. Sousa HW, Silva JL, S Neto M. A importância do profissional farmacêutico no combate à automedicação no Brasil. Rev elet farm. Imperatriz, 2008. 5(1): 67-72.

10. Rozenfeld S. Prevalência, fatores associados e mau uso de medicamentos entre os idosos: uma revisão. Cad. Saúde Pública. Rio de Janeiro, 2003 maio-jun;19(3):717-724.

11. Hobson M. Medication in older patients. West J Med. 1992. 157(5):539-543.

12. Gallagher P, Barry P, O'mahony D. Inappropriate prescribing in the elderly. J Clin Pharrm Ther. 2007. 32(2):113-21.
13. Dicionário de especialidades farmacêuticas, 2012-2013. 41. ed. Rio de Janeiro: Epub; 2013. 940p.

14. Santello FH, Redigolo E, Toniello WMM, Monteiro SCM. Perfil da automedicação em idosos no município de Barretos/ São Paulo/ Brasil. Infarm Ciênc Farm. 2013. 25(1): 32-36.

15. Almeida JPG, Cantuária BA, Assis JR. Automedicação realizada pelos pacientes idosos do NASPP em Montes Claros - MG. Rev Mult Faculd Integradas Pitágoras de Montes Claros. 2012 dez; 10(15): 94-103.

16. Galato D, Madalena J, Pereira GB. Automedicação em estudantes universitários: a influência da área de formação. Ciênc Saúde Col. 2012. 17(12):3323-3330.

17. Nascimento JP, Valdão GBM. Automedicação: educação para prevenção. In: Anais da Conferência Internacional de Estratégia em Gestão, Educação e Sistemas de Informação, 2012; Góias. Goiânia, 2012.p. 813.

18. Linjakumpu T, Hartikainen S, Klaukka T, Veijola J, Kivelä SL, Isoaho R. Use of medications and polypharmacy are increasing among the elderly. J Clin Epidemiol. 2002. 55(8):809-17.

19. Oliveira MA, Francisco PMSB, Costa KS, Barros MBA. Perfil da automedicação em idosos residentes em Campinas- São Paulo. Cad. Saúde Pública, Rio de Janeiro, 2012. 28(2):335-345.

20. Bortolon PC, Medeiros EF, Naves JOS, Karnikowski MGO, Nóbrega OT. Análise do perfil da automedicação em mulheres idosas brasileiras. Ciênc Saúde Coletiva. 2008. 13(4):212-26.

21. Musial DC, Dutra JS, Becker TCA. Automedicação entre os brasileiros. SaBios-Rev Saúde e Biol. 2007. 2(2): 5-8.

22. Schmid B, Bernal R, Silva NN. Automedicação em adultos de baixa renda no município de São Paulo. Rev. Saúde Pública. 2010. 44(6):1039-1045.

23. Souza, AC. Como manda o figurino: práticas terapêuticas entre idosos de Porto Alegre [Dissertação]. Porto Alegre: Escola de Enfermagem, Universidade Federal do Rio Grande Sul; 2005. 115p. 